\title{
El Programa de desarrollo y paz del Magdalena Medio: \\ ¿un modelo de construcción de \\ paz para el post conflicto en \\ Colombia?*
}

\section{The Magdalena Medio Peace and Development Programme: a Peacebuilding Model for Colombia's Post Conflict?}

Miguel Barreto Henriques ${ }^{* *}$

Recibido: 17/03/2014

Aprobado: 14/07/2015

Disponible en línea: 30/11/2015

\section{Resumen}

Este artículo incide sobre una experiencia sui generis de construcción de paz en Colombia, el Programa de desarrollo y paz del Magdalena Medio (PDPMM). El objetivo principal es evaluar en qué medida el PDPMM se concibe como un verdadero 'laboratorio de paz' y abre caminos novedosos y 'fórmulas' alternativas para la paz a

\begin{abstract}
This paper focuses on a sui generis peacebuilding experience in Colombia - the Magdalena Medio Peace and Development Programme (PDPMM). Its main purpose is to assess the extent to which the PDPMM stands as a true 'peace laboratory' and paves new roads and alternative 'formulas' for peace at local and regional levels, which could
\end{abstract}

doi:10.11144/Javeriana.papo20-2.pdpm

${ }^{*}$ Artículo de Investigación, Este documento recoge algunos de los insumos e ideas clave de la tesis doctoral "Laboratorios de Paz en territorios de violencia(s): ¿abriendo caminos para la paz positiva en Colombia?", presentada en la Universidad de Coimbra, Portugal, y del proyecto de investigación "Experiencias de Paz: lecciones aprendidas para Colombia - presencia integral del Estado en el territorio (PIET)", desarrollado en la Universidad Jorge Tadeo Lozano de Bogotá.

** Licenciado en Relaciones Internacionales y Doctor en Política Internacional y Resolución de Conflictos de la Universidad de Coimbra, Portugal, donde presentó la tesis "Laboratorios de Paz en territorios de violencia(s): ¿abriendo caminos para la paz positiva en Colombia?", premiada por la Casa de la América Latina/Banco Santander como mejor tesis en ciencias sociales y humanas en 2013. Ha sido investigador del Centro de Recursos para Análisis de Conflictos (CERAC) en Bogotá (2007-2009), del CEUNEUROP (Centro de Estudos da União Europeia da Faculdade de Economia da Universidade de Coimbra) (2006-2007), y del NICPRI (Núcleo de Investigação em Ciência Política e Relações Internacionais (2008-2012), en Portugal. Desde el 2012 se encuentra vinculado , como Profesor Asociado al Programa de Relaciones Internacionales de la Universidad Jorge Tadeo Lozano y como investigador al Observatorio de Construcción de Paz. Director del Observatorio de Construcción de Paz. Correo electrónico: miguel.barretod@utadeo.edu.co 
nivel local y regional, que se podrían replicar en otras regiones del país, en un potencial escenario de post-conflicto colombiano. La hipótesis de trabajo es que el PDPMM se configura como un modelo consolidado y válido de construcción de paz positiva a nivel regional, de lo cual se pueden rescatar diversas enseñanzas y 'buenas prácticas' para la construcción de una paz sostenible y duradera en Colombia.

\section{Palabras clave}

construcción de paz; post conflicto; programas de desarrollo y paz; Programa de desarrollo y paz del Magdalena Medio; paz positiva; paz sostenible; cultura de paz

\section{Cómo citar este artículo:}

Barreto-Henriques, M. (2015). El Programa de desarrollo y paz del Magdalena Medio, ¿un modelo de construcción de paz para el post conflicto en Colombia? Papel Político, 2O(2), 461-479. http://dx.doi.org/10.11144/ Javeriana.papo20-2.pdpm be replicated in other regions of the country, in a potential post-conflict scenario in Colombia. The hypothesis of the present work is that the PDPMM represents a valid and consolidated positive peacebuilding model at the regional level, from which various lessons can be learned, in order to build a sustainable and lasting peace in Colombia.

\section{Keywords}

peacebuilding; post conflict; Peace and Development Programmes; Magdalena Medio Peace and Development Programme; positive peace; sustainable peace; culture of peace 


\section{Introducción}

Actualmente Colombia se enfrenta a uno de los mayores desafíos de su historia reciente: poner definitivamente fin a un sangriento conflicto armado de cinco décadas y construir un país y una sociedad en paz. La posibilidad de un acuerdo político entre las FARC y el gobierno nacional en la Habana permite soñar con ese escenario, pero también genera varios interrogantes: ¿Cómo será la etapa del post conflicto en Colombia? ¿Qué desafíos enfrentarán el país y la sociedad? ¿Qué riesgos y peligros podrían afrontar? ¿Cómo se pacificará política, social y culturalmente la sociedad y las regiones? ¿Cómo se procesará la construcción de una paz duradera y sostenible a largo plazo? ¿Qué modelo de paz se debe seguir? ¿Qué actores podrán desempeñar un papel importante en este cuadro?

El presente documento se enfoca en el Programa de desarrollo y paz del Magdalena Medio (PDPMM), una iniciativa de paz de la sociedad civil puesta en marcha en una de las regiones más conflictivas del país. Pretende analizar en qué medida el modelo de construcción de paz puesto en práctica en esta región encierra elementos válidos para un escenario de post conflicto en Colombia, buscando identificar buenas prácticas para la construcción de una paz positiva y duradera en el país.

\section{Los retos del post conflicto en Colombia}

Si algo nos han demostrado las experiencias de varios países en el mundo, como El Salvador, Guatemala o Irlanda del Norte, es que un escenario de post conflicto trae tantos desafíos políticos y sociales a un país como el mismo proceso de paz y el conflicto armado. Un proceso de negociación y un acuerdo de paz tienen un alcance limitado. Permiten un primer paso fundamental e indispensable para la paz: el silenciamiento de los fusiles de los actores armados, pero no necesariamente la eliminación de los gérmenes de violencia y las raíces de la conflictividad. Inciden tan solo sobre una de las dimensiones de un conflicto - los aparatos armados de los grupos ilegales y sus comandos políticos (Granada, Restrepo, \& Vargas, 2009, p. 103). Por lo tanto, no son garantía de un país pacificado, sino de una paz frágil, limitada e imperfecta. No generan una paz sostenible y duradera que sea transversal a todos los niveles y esferas de relaciones sociales.

Colombia es un país cuya violencia es un fenómeno particularmente complejo y multidimensional. Adopta diversas modalidades, formas y expresiones en las cuales se interconectan y cruzan la violencia insurgente, paramilitar, mafiosa, criminal y narcotraficante, y no se circunscriben a la existencia de grupos alzados en armas (McDonald, 1997, p. 1). La violencia en Colombia está enraizada y es alimentada y sostenida por factores políticos y socio-económicos estructurales.

El conflicto armado está enmarcado en procesos históricos de largo plazo y de varias índoles que se tradujeron en un panorama de marginalización política, social y económica de amplios sectores de la población colombiana, en particular, del campesinado y en la 
exclusión o precaria integración de algunas regiones y zonas de Colombia, del desarrollo, la democracia y las instituciones del Estado. Se cristalizó una brecha entre el centro y periferia del país, entre ‘dos Colombias', asimétricas política, social y económicamente, factor que ha alimentado históricamente el desarrollo de grupos armados ilegales y la emergencia de violencia bajo distintas modalidades y formas (Barreto, 2012). La violencia armada emergió sociológicamente y se ha circunscrito sobre todo en la Colombia rural.

Por lo tanto, si los esfuerzos de pacificación de Colombia se limitan a la dimensión de meros acuerdos entre el Estado y la insurgencia, y no se abordan las causas de la conflictividad, hay un gran riesgo de que el país no sea verdaderamente pacificado y que la violencia reincida o se transfigure, y asuma nuevas formas y modalidades en los territorios periféricos y rurales del país, y se sigan generando y alimentado grupos con la capacidad de desafiar el monopolio legítimo de la fuerza, sea en la forma de guerrillas, grupos paramilitares, narcotraficantes o pandillas. De hecho, el riesgo de no abordar las causas subyacentes de los conflictos es tan grande como no tratar los síntomas de una enfermedad (Burton, 1990).

El fenómeno de las ‘nuevas bandas emergentes' o BACRIM, como las Águilas Negras, los Rastrojos y los Urabeños, que sucedieron el proceso formal de desmovilización de las AUC, o de las maras en Centroamérica, en donde una "guerra de frentes se convirtió en una guerra de todas las esquinas" de acuerdo a Vincenti, ex coordinador de la línea 1 de la Asistencia Técnica Internacional al Segundo Laboratorio de Paz (entrevista, febrero 26 de 2008), en gran medida revela esta realidad y constituye una alerta para los peligros y riesgos de un escenario post-negociación.

Por esta razón, es importante subrayar que la paz no se materializará en Colombia, como un 'café instantáneo', el día que se firme un acuerdo entre el gobierno y las FARC. La construcción de una paz duradera, sostenible y positiva ${ }^{1}$ implica un proceso mucho más largo y que requiere la participación de muchos más actores de la sociedad, más allá del Estado y de los actores armados.

Pasará por las distintas esferas y niveles de la organización social, por las políticas macro definidas desde la Casa de Nariño en Bogotá, pero también por cada región, territorio y vereda del país, que tendrán que generar nuevas formas de convivencia.

\footnotetext{
${ }^{1}$ El concepto de paz positiva acuñado por Johan Galtung (1996), amplió el significado convencional de la paz como antítesis de la guerra y revaluó el concepto de violencia de su sentido y connotación tradicional -violencia física- para darle una significación más amplia. Desde este punto de vista, la paz no es solamente la ausencia de la guerra, estos no son elementos equivalentes. Lo que le anima es una visión de la paz, no como antónimo de la guerra, sino de violencias. Galtung concibe una paz definida positivamente, que implica una reestructuración profunda de las relaciones humanas. Transmite un concepto amplio y denso de paz, uno que implica mucho más que el silenciamiento de los fusiles. Se basa en una visión integral de la paz y los conflictos que establece un vínculo claro y fuerte entre el desarrollo, la justicia social y los temas de la paz.
} 
Implica un 'desarme' social, político, económico y cultural de la sociedad colombiana, y el cambio de una cultura de violencia por una cultura de paz. Es un proceso paulatino y progresivo que se juega a varias escalas y niveles e implica la participación amplia de la población. Usando una metáfora de Lederach (1997, p. 37), uno de los más reputados teóricos en temas de resolución de conflictos para construir la 'casa de la paz', se requiere un trabajo tanto en el techo como en los cimientos, tanto en la cima, como la base de la pirámide del conflicto; todos los niveles son igualmente importantes para que esta se sostenga y no caiga.

\section{El Programa de desarrollo y paz del Magdalena Medio}

El Programa de desarrollo y paz del Magdalena Medio figura como una de las más emblemáticas experiencias de paz que han emergido desde la sociedad civil en Colombia en los últimos 20 años. Su singular enfoque y filosofía y su labor junto a las comunidades de la región a favor de la paz, el desarrollo y la 'democratización de la democracia local', lo han transformado en un referente y un modelo de construcción de paz desde la base, que se ha replicado en varias regiones del país a través de otros programas de desarrollo y paz (PDP).

Este programa nació a mitad de la década de 1990 en una de las zonas más conflictivas del país en un contexto de crisis social y económica y de agudización del conflicto y su impacto sobre la población civil en el Magdalena Medio (Molano, 2009). Se pueden identificar fundamentalmente dos factores y propósitos en su origen: por un lado, se le pretendía asumir como una forma de resistencia civil y de búsqueda de oxígeno en medio de la violencia; mientras que el otro resultó de una confluencia de intereses y preocupaciones éticas, políticas y sociales entre la compañía de petróleo Ecopetrol, su sindicato laboral (USO) y la Diócesis de Barrancabermeja en torno a una de las contradicciones esenciales del Magdalena Medio: ¿jpor qué una región tan rica² tiene tanta pobreza y violencia?

Pretendían diagnosticar las causas de la violencia y la pobreza en la región y visualizar posibles soluciones y vías para la paz y el desarrollo. En este ámbito, se conformó el PDPMM en 1995 como programa para promover dinámicas de paz a través de la promoción de procesos de participación y organización popular y del fomento de procesos socioeconómicos de desarrollo sostenible e inclusivo (CDPMM, 2005). desde entonces, el PDPMM se ha consolidado como un programa multidimensional de construcción de paz desde la base, configura un amplio conjunto de procesos sociales, culturales, económicos y políticos en el ámbito local y regional. Busca construir la paz en sus múltiples aspectos e incidir sobre las distintas facetas de la violencia.

${ }^{2}$ La producción, refinación y transporte de petróleo y gas originaron en el 2005, el $88 \%$ del producto bruto regional, correspondiente a cerca de 3.696 millones de dólares (Castilla, 2007). 
Pero sobre todo, el PDPMM ha puesto énfasis en el desarrollo como medio para la paz. Parte del principio de que la construcción de la paz pasa por generar nuevas condiciones de vida en el campo, la inclusión de los campesinos y el desarrollo socioeconómico de las comunidades (Saavedra \& Ojeda, 2006). Su lectura política del conflicto colombiano define elementos como la pobreza, la inequidad y la exclusión socioeconómica como causas estructurales del conflicto y de la violencia. Por lo tanto, prescribe el desarrollo incluyente y sostenible como medio y receta para la paz. Sobre estos dos ejes se soportan líneas conceptuales y vectores de trabajo que pretenden propiciar modelos y caminos socioeconómicos distintos, más inclusivos y equitativos, y que fomenten tanto un desarrollo humano y sostenible, como la construcción de una paz duradera y estable.

Por encima de todo, el PDPMM es un intento de generar condiciones sociales, económicas, políticas y culturales para la paz en territorios marginados y periféricos. El principal objetivo de esta experiencia es la eliminación de las causas de raíz del conflicto a un nivel micro -en particular la exclusión social, económica, política y regional, mediante la búsqueda de integración de los sectores sociales tradicionalmente marginados de la población colombiana, como los campesinos, los jóvenes, los pescadores, los mineros, las mujeres, y su acercamiento a la institucionalidad, al desarrollo y a la democracia (Barreto, 2012).

La filosofía del PDPMM puesta en marcha en sus procesos de base, se sostiene en una metodología participativa. Intenta construir plataformas de actores sociales con los sectores excluidos de la población para dar voz a los que no tienen voz, alentándolos y ayudándolos a construir propuestas sociales, económicas y políticas alternativas. Considera que estos no son solo las principales víctimas de la violencia en Colombia, sino también actores esenciales para la construcción de un país en paz (Barreto, 2012, p. 293).

El PDPMM es así, principalmente un modelo de intervención territorial, de construcción de la paz desde las dinámicas de la región y a partir de la participación de las comunidades y actores del territorio. Configura un modelo regional de construcción de paz y de descentralización de la transformación del conflicto (Barreto, 2009, p. 559).

El liderazgo de la iniciativa ha pertenecido desde su origen a la iglesia católica, particularmente a sectores progresistas de los jesuitas influenciados por la teología de la liberación. El padre Francisco De Roux, hoy provincial de la Compañía de Jesús en Colombia, fue el mayor ideólogo del programa y director -durante varios años- de la Corporación de Desarrollo y Paz del Magdalena Medio (CDPMM), una entidad privada conformada por el CINEP y la Diócesis de Barrancabermeja que funciona desde 1998 (Molano, 2009), orienta, dinamiza y coordina el PDPMM y funciona como su guía, orientador estratégico y agente técnico y administrativo (CDPMM, 2005). 


\section{El modelo de construcción de paz del PDPMM y su potencial para un escenario de post conflicto en Colombia, buenas prácticas para la paz positiva y duradera}

La posibilidad de emergencia de un escenario de post conflicto formula profundos desafíos para Colombia. A la firma de un acuerdo se sucederá una larga y exigente etapa de construcción de la paz en el largo plazo que implica la participación de amplios sectores de la sociedad colombiana y la incidencia sobre distintas dimensiones de la conflictividad.

En este marco, el PDPMM podría adquirir un significado e importancia adicionales en cuanto a modelo consolidado de construcción de paz positiva, potencialmente aplicable en un escenario de post conflicto en Colombia.

El potencial de su modelo y enfoque para la paz, reside en varios factores y elementos que se han desarrollado e implementado en casi 20 años de labor para la paz junto a las comunidades. El PDPMM, tanto en su filosofía de paz como en sus procesos sociales, ha generados diversas metodologías e instrumentos en áreas como el desarrollo rural, la organización comunitaria, el fortalecimiento institucional, la democratización de la vida política y la generación de una cultura de paz, que podrían ser replicadas en escalas más amplias en un escenario de post conflicto en Colombia, con vista a la construcción de una paz sostenible y duradera.

En seguida, buscaremos identificar algunas de las 'buenas prácticas' del PDPMM en términos de construcción de paz que configuran enseñanzas para la búsqueda de una paz positiva en Colombia.

\section{El PDPMM como modelo de intervención territorial en las zonas rurales}

El PDPMM ha diseñado y consolidado un modelo de construcción de paz positiva y sostenible desde la base, puesto en marcha a partir de la especificidad de la región del Magdalena Medio. Configura un modelo de intervención territorial y de fomento de desarrollo rural para las zonas periféricas de Colombia, espacios geográficos y sociales que han alimentado históricamente el conflicto. Muestra líneas de acción concretas para la construcción de la paz duradera en estos territorios marginados del país, teniendo en cuenta los factores estructuradores del conflicto en Colombia, estableciendo una metodología válida de aproximación a las causas profundas del conflicto y las estructuras de exclusión que impiden el ejercicio de la ciudadanía para una buena parte de la población. Ha abierto una vía regional para la paz y el desarrollo en el país a partir de procesos sociales micro que descentralizan la construcción de paz en Colombia (Barreto, 2009, p. 559). Consolida una mirada y una propuesta de región e integra a la población vulnerable y al campesinado.

Estos elementos son fundamentales para forjar un proceso integral de construcción de paz en el posconflicto, pues una política o un proceso de paz en Colombia que no 
tenga en cuenta la variable territorial y las particularidades regionales, está condenada a fracasar o a obtener impactos mitigados. Para que sea sostenible y duradera no puede cimentarse en un enfoque estrictamente nacional. Necesita incluir escalas y enfoques espaciales distintos, agendas locales y regionales de paz, pasando por supuesto por el involucramiento de las regiones, la participación activa de las comunidades y de la sociedad civil organizada a partir de los territorios, de forma que estos puedan desactivar expresiones violentas y enraizar dinámicas de resolución pacífica de conflictos, de cultura de paz y de inclusión política, social, económica y regional.

El PDPMM, tal como los demás PDP en Colombia, permiten soñar, discutir y construir la paz desde las bases, con las comunidades locales, lo que en última instancia, ayuda a edificar los pilares de una Colombia en paz. Muestran que las diferencias se pueden dirimir a través del diálogo, lo que configura en sí mismo la esencia de la resolución de los conflictos, pero también la médula de una democracia. Por lo demás, desarrollan instrumentos para que estas mismas comunidades dialoguen y se articulen con las instituciones locales y nacionales, lo que resulta cardinal para la implementación de políticas de paz.

\section{Un modelo integral de construcción de paz}

La principal fortaleza y singularidad del modelo de construcción de paz del PDPMM reside en la integralidad y multi-dimensionalidad de su enfoque. Integra simultáneamente en sus procesos sociales, diversas áreas y componentes de la construcción de la paz, desde la vertiente socio-económica a la cultural, de los derechos humanos a la participación social, de la dimensión ambiental a la institucional. Entre sus iniciativas se incluyen proyectos productivos, programas de educación y arte para la paz, procesos de resistencia civil, iniciativas de planeación participativa, redes de organización comunitaria y canales de diálogo y articulación entre la sociedad civil y el Estado. Constituyen una iniciativa de aproximación integral y holística para la paz que tiene como horizonte la construcción de la paz positiva y no solo la ausencia de la guerra. Esta, es a la vez una exigencia y el mayor reto de un escenario de posconflicto: pacificar el país trasversal e integralmente en sus diversos campos, sectores y regiones, más allá de la violencia armada y de los grupos armados ilegales.

En juego está el generar relaciones sociales más pacíficas, mecanismos de resolución no violenta de controversias, reconciliar comunidades y grupos sociales antagónicos, transformar una cultura de violencia en una cultura de paz y direccionar las actividades económicas hacia la paz y la legalidad. Todos estos aspectos son los que permiten generar una paz sólida, duradera y sostenible. Es un proceso que pasa necesariamente por políticas públicas de paz, integrales y multidimensionales que incidan sobre los distintos aspectos de la conflictividad y los sectores y grupos sociales afectados por el conflicto a lo largo del territorio nacional, pero que exigen de igual forma la participación activa de la sociedad civil. 
El PDPMM asumió esta realidad, comprendiendo que la construcción de la paz se hace en varios frentes. Esta es una de los mayores enseñanzas que deja a Colombia y que sería importante replicar a nivel nacional en un escenario de post conflicto en el país.

\section{El desarrollo como medio para la paz}

Otro de los elementos fundamentales del modelo de construcción de paz del PDPMM que figura como una buena práctica para el post conflicto en Colombia, se relaciona con su perspectiva de conjugar la paz con el tema del desarrollo. Esta ha sido una de las propuestas más emblemáticas del PDPMM, constituyendo la fuerza motriz y corazón de sus procesos sociales. Construir la paz mediante procesos de inclusión económica y de desarrollo humano sostenible, aparece de forma innovadora e indisociable en el PDPMM. Fue pionero en Colombia en su intento de combinar y 'casar' la construcción de paz desde la base con procesos de desarrollo.

Según Restrepo, economista, director del Centro de Recursos para Análisis de Conflictos (CERAC), profesor de la Universidad Javeriana, es una 'intervención dual' (entrevista, diciembre 7 de 2008), como queda claro y transparente en la designación Programa de Desarrollo y Paz. Emerge como una propuesta y una iniciativa que se propone construir simultáneamente desarrollo y paz de modo integral, como una alternativa de legalidad a la depredación de recursos y a la violencia, lo que imprime una dinámica multidimensional al PDPMM en la persecución de la paz.

En el PDPMM hubo una convergencia entre estas dos nociones y vías. El desarrollo se instituye como un incentivo y un medio para la construcción de la paz. La intencionalidad es manifiestamente política. El énfasis no está en la reducción de la pobreza ni en la obtención de beneficios económicos, sino en el problema del conflicto y de la violencia y en la generación de condiciones de paz y convivencia. El centro y horizonte es la paz. Se pretende en esencia, que los procesos de desarrollo sean un factor que saque espacio y pobladores del conflicto. Si hay una intervención desde lo económico, es porque las condiciones de desarrollo son vitales para una vida digna, sin la cual, no puede haber una paz estructural y positiva.

Hay una originalidad en la visión y estrategia de integralidad del PDPMM. Es una intervención sui géneris que permite integrar la población civil en espacios y procesos económicos, pero que al mismo tiempo reduce la inseguridad y abre horizontes de paz, pues al generar ingresos y mejorar las condiciones de desarrollo, crea oportunidades y capacidades para auto determinarse y tener una mayor participación política, produce a su vez, sensaciones de bienestar, así como proporciona un desincentivo para la asunción de riesgos criminales y violentos (Barreto, 2012).

Además de un modelo de construcción de paz, el PDPMM es una propuesta y estrategia de desarrollo humano e incluyente direccionado a territorios rurales, que se pone 
en práctica mediante proyectos y procesos de varias índoles, como las fincas campesinas, programas de crédito asociativo y microcrédito, iniciativas de economía popular y programas de educación y formación direccionados a zonas rurales.

Por esta razón, en la mayoría de los proyectos, procesos e iniciativas del PDPMM se vincula la dimensión social a la económica. El trasfondo es una generación de capacidades, el fortalecimiento organizativo y un cambio cultural (Briceño, 2007). Los proyectos económicos son intervenciones que buscan generar escenarios de participación y decisión colectiva, fomentar el diálogo y las alianzas entre diferentes sectores de la sociedad civil y un nuevo entendimiento de cómo acercarse a las autoridades regionales y nacionales. Se conciben como procesos de inclusión de grupos sociales y comunidades pobres y marginadas en términos económicos, pero también con repercusión social, política y cultural. Tienen en vista la posibilidad de entrada de estos grupos a los circuitos económicos del mercado, pero también al espacio público.

Esto figura como un enfoque y metodología valiosos en el marco de un proceso de construcción de la paz a largo plazo en Colombia. Generar dinámicas de desarrollo humano que abarquen e integren a la totalidad de la población colombiana, constituye un reto inmenso y un requisito y condición indispensable para construir una paz duradera en el país. La construcción de la paz pasa por generar nuevas condiciones de vida en los territorios rurales de Colombia. Efectivamente, la inclusión socioeconómica de los campesinos se concibe como esencial, en tanto que la falta de horizontes de vida de la gente en el campo es un factor determinante que no solo ha alimentado históricamente el conflicto armado, sino que ha empujado a millares de familias hacia la economía de la coca.

La firma de un acuerdo de paz no borra esta realidad, ni esta amenaza y germen de violencia. Este es un sector social que seguirá en una posición delicada y de riesgo en un escenario de post conflicto. Asimismo, el narcotráfico y la economía de la coca, a pesar de su vínculo y ‘casamiento’ con el conflicto y los grupos armados en los últimos veinte años, constituyen fenómenos que transcienden el conflicto armado y que les sobrevivirán, siguiendo al tema de la seguridad en Colombia, que tocará tener en consideración.

Por consiguiente, estos son elementos y fenómenos que es necesario asumir y abordar en un escenario de post conflicto en Colombia para construir una paz sostenible y duradera y prevenir una transfiguración de la violencia.

Diseñar y generar alternativas de vida y de ocupación económica en el campo son formas de retirar espacio social a la violencia armada y a la economía ilegal. Contribuyen para inmunizar sectores sociales -como los campesinos y los jóvenes-, disminuyendo los riesgos de asunción de actividades violentas y criminales. Efectivamente, la intervención sobre las raíces de la conflictividad y violencia es lo que en última instancia, sentará las raíces de una Colombia verdaderamente pacificada. 
El PDPMM ha tenido siempre este horizonte en vista. Ha puesto en evidencia la importancia de generar instrumentos de promoción de desarrollo rural integral y procesos de inclusión de los campesinos en la vida social, económica, política y productiva, dando nuevas posibilidades de vida a varios sectores sociales, como los jóvenes de las comunas de Barrancabermeja o los campesinos del sur del Bolívar. Su enfoque, al direccionarse a las causas profundas de la violencia en los territorios que históricamente han alimentado la violencia armada, ha abierto caminos para una paz positiva y ha generado bolsas de paz a nivel micro que sirven como aprendizajes y pistas para un país en busca de la pacificación.

\section{Instrumentos de integración de grupos sociales marginados y de riesgo}

Además del campesinado, hay otros grupos sociales marginados y de riesgo en Colombia que necesitan ser integrados a nivel político, socioeconómico y regional para construir una paz sostenible y duradera.

El trabajo con los jóvenes es particularmente importante en este contexto. En términos sociológicos, la violencia armada, sea política o criminal, es producto, mayoritariamente, de jóvenes del sexo masculino. Son la carne de cañón y la materia prima de la violencia que alimenta los actores de la guerra.

En esta medida, el trabajo del PDPMM en el sentido de conferir ocupación y formación para los jóvenes y de proveer alternativas de vida para este sector social (en específico a los sectores más excluidos), constituye un medio directo que toma espacio social frente a la violencia armada y edifica los cimientos para la paz directa y positiva.

El PDPMM se ha instituido como una plataforma de inclusión de grupos sociales marginados y excluidos, como campesinos, jóvenes, obreros y mujeres. Ha desarrollado y puesto en práctica diversos mecanismos, programas y procesos de integración de estos sectores en el ámbito socioeconómico, político, cultural y simbólico, mediante instrumentos como el arte, la educación, el deporte y la organización social y comunitaria.

A través de la participación social y cultural de los jóvenes, el involucramiento en grupos de canto y baile, la práctica deportiva, entre otros, se inspira el amor a la vida y se rescatan jóvenes del conflicto. Como reconoce Mayerly Méndez, una de las integrantes de la Red de Jóvenes del Magdalena Medio,

[...] solamente el hecho de que jóvenes estuvieran en una organización, que se sentaran a hablar, a hacer amigos y a compartir su tiempo, ya eso generaba que la guerra y los actores armados desaparecieran como opción de vida. (entrevista, septiembre 2 de 2008)

En juego está la multiplicación de las opciones de cada uno, visto como un proceso conducente al desarrollo humano y el refuerzo de lo que Francisco De Roux (entrevista, 
enero 23 de 2008) generalmente llama de la 'vida querida'. Son formas de apartar a estos grupos sociales de actividades criminales y violentas, pero también de generar una cultura de paz. Se busca construir la paz a partir de las mentes de los jóvenes, creando condiciones culturales y económicas para que estos generen otros proyectos de vida y se alejen de opciones de riesgo como los cultivos de uso ilícito o la integración a los grupos alzados en armas.

Efectivamente, la construcción de la paz exige crear condiciones para prevenir el involucramiento de los jóvenes y otros grupos sociales de riesgo en este tipo de actividades, de forma a que no ocurra una 'centro-americanización' del post conflicto en Colombia y se reincida en la violencia bajo otras formas y modalidades. En ese ámbito, las iniciativas y procesos del PDPMM en el sentido de integración de los jóvenes, constituyen ejemplos para abordar este tema, con miras a la construcción de una paz sostenible y duradera en el país.

\section{La construcción de una cultura de paz}

La construcción de la paz tiene una fuerte dimensión cultural. En última instancia, pasa por pacificar las mentes y por trabajar con todos los actores y en todas las esferas, en favor de posturas más tolerantes, civilizadas y democráticas. Es un proceso social, pero también personal y sicológico, como queda claro en el principio proclamado por la Organización de las Naciones Unidas para la Educación, la Ciencia y la Cultura (Unesco) de que si las guerras nacen en la mente de los hombres, es en la mente de los hombres que se deben construir los baluartes de la paz (Pureza, 2001).

El PDPMM ha realizado una labor extraordinaria en este campo y muchos de sus elementos e iniciativas son rescatables puesto que promueven y desarrollan varios proyectos que hacen énfasis en una cultura de paz. Por medio de una educación para la paz y los derechos humanos, el empleo de programas y talleres cívicos, actividades deportivas, iniciativas artísticas ${ }^{3}$ y radios comunitarias ${ }^{4}$ direccionadas para la paz, la ciudadanía, los

\footnotetext{
${ }^{3}$ Corresponden a iniciativas que no trabajan el arte por el arte, es decir en cuanto expresiones estrictamente artísticas, sino en tanto medios de promoción de procesos culturales con énfasis en la paz y los derechos humanos. Buscan transformar a las personas en sujetos de paz en su dimensión intrapersonal e interpersonal. Como señala Guido Ripamonti, director del grupo de teatro del Centro Cultural de la Ciudadela Educativa: "empezamos por el teatro, pero les mostramos que no estaban aquí para hacer teatro en sí mismo, sino para transformar al ser humano y construir gestos de paz" (entrevista, junio 26 de 2013).

${ }^{4}$ Las radios comunitarias figuran igualmente como un instrumento valioso de construcción de una cultura de paz. EI PDPMM ha apoyado el fortalecimiento de una red de radios comunitarias con enfoque en los territorios rurales, que, como señala su director, pretenden ser "la voz de los que no tienen voz" (Hoyos, entrevista, septiembre 10 de 2008), y un método de promoción de la participación pública y empoderamiento social de los campesinos. Se concibieron, más que como un vehículo cultural o un medio de difusión musical, como un proceso social y de construcción de lo público, en torno a temas como "la ciudadanía, el género, el medioambiente, las identidades culturales en la región, la niñez, la juventud, etc." (Hoyos, entrevista, septiembre 10 de 2008).
} 
derechos humanos (OPI, 2006). El PDPMM ha buscado construir un imaginario colectivo favorable para la paz. En juego está cambiar las formas de pensar y actuar que legitiman la violencia, la exclusión y la violación de los derechos humanos e inculcar valores proclives a la paz, como la tolerancia, el diálogo y la solidaridad. Según Vargas, sociólogo, investigador del CINEP y funcionario de la CDPMM, la meta es construir una estructura integral de paz, en la cual se incluyan tanto la dimensión económica y las instituciones políticas, como las personas y los paradigmas de pensamiento (entrevista, abril 23 de 2007).

En este contexto la educación para la paz resulta de gran relevancia. Las escuelas son un importante referente de valores y un instrumento crucial para la transformación de los conflictos (Saavedra \& Ojeda, 2006). Consciente de esto, el PDPMM ha desarrollado una estrategia pedagógica y variados proyectos en el campo educativo, como "Ciudadela educativa”, "Bio-pedagogía” y "Propuesta educativa de Barrancabermeja y Puerto Berrío". Estas escuelas de paz pretenden propiciar escenarios para el intercambio cultural, para incrementar la solidaridad, nutrir referentes simbólicos de paz y fortalecer una opinión pública sobre la paz (OPI, 2006). Asimismo, son medios para la transformación del conflicto en Colombia, pero también para las micro transformaciones de los conflictos cotidianos de la gente en una forma positiva, imaginativa y creativa, para fomentar el uso del diálogo en menoscabo de la violencia, de la negociación en detrimento de la confrontación y de tener actitudes, posturas y valores más proclives a la paz.

Estos procesos han permitido generar 'islas' de una cultura de paz, es decir, micro espacios y expresiones de paz en donde los valores de la civilidad y la solidaridad se imponen y se alejan de las dinámicas de la violencia. Han desarrollado un trabajo valiosísimo en el sentido de desarrollar y generar una nueva cultura en los territorios y veredas de las regiones y cambiar los paradigmas de pensamiento de las comunidades. Han fomentado la exploración y desarrollo desde lo cotidiano de nuevas formas de relación e interrelación que superan la polarización del conflicto armado, deslegitiman cultural y socialmente la guerra y el recurso a la violencia. Aquí la paz emerge como un proceso que se va construyendo cotidianamente desde las veredas de las regiones, corporizando una "paz del día a día" (Richmond, 2008, p. 109). Estos procesos paulatinos son los que permiten sentar los cimientos de una paz sostenible y duradera.

Las emisoras comunitarias se han convertido en instrumentos de participación e inclusión política y social de las comunidades de las veredas del Magdalena Medio. Actores tradicionalmente excluidos e invisibles, como los jóvenes, los campesinos y las mujeres, logran ser oídos y reconocidos, participar en la esfera pública y garantizar la visibilidad de sus pensamientos, de su condición y posición social por esta vía (CDPMM, 2005). Las radios comunitarias son la voz de los campesinos y las poblaciones de las veredas. Son una forma de llevar las periferias geográficas y sociales a los medios de comunicación dominados por las agencias hegemónicas urbanas. Se configuran como elementos constructores de inclusión política, social y cultural, lo que resulta fundamental en el panorama colombiano para la construcción de la paz positiva. 


\section{Procesos de democratización de la democracia a nivel local y acercamiento del Estado al ciudadano}

El PDPMM figura como un intento de democratización en varios niveles. Ha puesto en marcha diversos procesos y metodologías participativas, así como mecanismos de fomento de una democracia participativa ${ }^{5}$ local y regional, con miras a acercar a los ciudadanos a las instituciones y fomentar un nuevo modelo de relación entre gobernantes y gobernados. Estos instrumentos -asentados en la articulación entre la sociedad civil y las instituciones públicas- se erigen como elementos fundamentales para la transformación del conflicto en un país cuya democracia es limitada (Pécaut, 1992) y que sufre de exclusión política, hechos en los que se basa la violencia armada.

El PDPMM entiende que la construcción de la paz en Colombia pasa por la democratización de un país que ha sido históricamente excluyente, e incluso represivo, en términos políticos, económicos y regionales. Pasa también por el involucramiento de la totalidad del territorio nacional y de los sectores sociales de la población -desde los campesinos hasta los obreros- en los beneficios y dividendos de las instituciones y de la democracia de la nación. Se parte del principio y de la convicción de que la inclusión política y la construcción de la democracia en lo local contribuyen para desbloquear los espacios de la violencia y favorecer la resolución pacífica de los conflictos (PNUD, 2008).

Son varios los ejemplos de estas iniciativas en la región: núcleos de pobladores ${ }^{6}$, presupuestos participativos ${ }^{7}$, asambleas veredales, asambleas constituyentes munici-

\footnotetext{
${ }^{5}$ Por democracia participativa se entiende el sistema y la práctica política en la que los ciudadanos son parte central y nuclear de la gestión de la res publica, participan activamente y de forma directa en la vida política. Se distingue de la democracia estrictamente representativa en la medida en que el proceso de toma de decisiones tiene como base la participación pública y este ejercicio no se delega a un representante (Guarín, 2008). Implica un ideal participativo, una inclusión política que no es meramente formal, una extensión de la ciudadanía y contratos sociales más inclusivos que posibilitan una democracia de mayor intensidad (Sousa, 2003).

${ }^{6}$ Los 'núcleos de pobladores' son el ejemplo emblemático de este tipo de iniciativas. Originados en el proceso de diagnóstico participativo sobre las causas de la violencia y la pobreza en la región que dio origen al PDPMM, constituyen espacios populares de participación y ejercicios de planeación participativa en los cuales las organizaciones locales y pobladores de un municipio formulan diagnósticos regionales y establecen propuestas de paz y desarrollo, expresadas en las propuestas municipales, subregionales o regionales (Katz, 2004). Definen cómo ven los pobladores a la región, cuáles son sus necesidades y qué quiere la comunidad para la región. Los núcleos de pobladores son la base del PDPMM y su fuerza motriz. Representan una herramienta fundamental de organización y participación ciudadana a través de la cual se eligen proyectos estratégicos para la vida social y económica de los municipios, se priorizan iniciativas comunitarias, se canalizan recursos y se establece la interacción y articulación con las administraciones locales (Barreto, 2012) ${ }^{7}$ Los presupuestos participativos corresponden a un mecanismo de planeación participativa que popularizó la alcaldía de Porto Alegre en Brasil y que se extendió a varios rincones del mundo, entre ellos Colombia. Mediante ellos los ciudadanos de un municipio deciden de forma directa
} 
pales, espacios humanitarios, equipos técnicos pedagógicos, consejos territoriales de planeación, 'trochas ciudadanas', entre otros. Su designación y contenido depende de la particularidad de cada proceso e iniciativa. Lo que tienen en común y que figura como una buena práctica, es que buscan que cada territorio genere ambientes de participación popular en donde la comunidad pueda tener voz, se empodere, priorice temas, haga propuestas políticas y dialogue con las instituciones, haciéndose parte activa de la gestión de la res publica. Son ejercicios democráticos con vista a atraer a la gente al centro de las decisiones políticas y de las políticas públicas, así como a fomentar una ciudadanía activa y una democracia incluyente (Villamarín, 2005).

Dichas iniciativas se fundamentan en la convicción de que para abordar la violencia y la pobreza hay que apoyar a la población en la concepción de sus propios proyectos de desarrollo y en las políticas públicas de forma directa y sin intermediación o tutelaje de los partidos políticos, dominados por lógicas clientelistas y corruptas (Molano, 2009). Entienden la participación como parte del proceso de desarrollo y de construcción de paz (García \& Sarmiento, 2002) y como antídoto para construir esperanza en medio de la barbarie y la violencia.

Estas iniciativas han adquirido gran importancia y han traído frutos políticos significativos. En esencia, han contribuido a la integración de poblaciones y comunidades históricamente excluidas de la vida política local, mediante su inclusión en la definición de las políticas públicas. Son instrumentos válidos y buenas prácticas de democratización de la vida política local y regional. Procesos como estos contribuyen al acercamiento de los ciudadanos a las instituciones y a poner a la población en el centro de la decisión política. También permiten una participación popular en el espacio público que favorece modos alternativos de hacer política en una región y un país en donde gran parte de la población se encuentra al margen de las instituciones y los procesos democráticos. Estos procesos han hecho un aporte a nivel local y regional para mejorar una democracia imperfecta, democratizar las instituciones, las prácticas y la cultura política, construir ciudadanía y superar el bipartidismo y clientelismo que históricamente han estado al servicio de la élite (ECP, 2006).

sobre parte del presupuesto público (Romero \& Navarro, 2010). Se apoya en la definición colectiva de las prioridades políticas de un municipio, a las cuales la alcaldía debe asignar recursos. Con los presupuestos participativos se pretende acercar la población y los ciudadanos a la gestión pública, confiriendo mayor acceso de las comunidades a las decisiones administrativas e imprimir más transparencia y ética en el manejo de los recursos públicos. Son formas de democratización del poder, de fortalecimiento de la participación ciudadana y de integración de la población en la vida política local. Este modelo se ha aplicado en varios municipios del Magdalena Medio como Barrancabermeja, Puerto Berrío, Bolívar, El Peñón, Cantagallo, Sabana de Torres, San Pablo Betulia y San Vicente del Chucurí, en varios casos con la promoción y el respaldo del PDPMM. 
En este orden de ideas, dichos procesos aportan a la transformación de la población en sujetos políticos, actores de su propio desarrollo, partícipes de la gestión de los municipios y de la res publica, y por ende, a la transformación del Estado y las instituciones locales. Se podrían percibir como referentes y como la incubadora de un nuevo modelo de relación entre el poder y los ciudadanos, que supere la desconfianza de la gente hacia las instituciones y el Estado, construyendo un sentido de autoridad legítima en la región. Es un trabajo de raíz en el sentido de democratizar la democracia (Sousa, 2003), de modificar tanto el contenido de las políticas públicas locales y regionales, como también el proceso de construcción y la toma de decisiones. Lo anterior configura un ejercicio de construcción del Estado social de derecho en el contexto local y regional, elemento de gran importancia política para el país. Cualquier proceso de construcción de paz, reconciliación y transición post-bélica implica algún grado de state-building.

El Estado se tiene que transformar y adaptar a un nuevo contexto de paz para construir una nueva sociedad y un nuevo país. Por lo demás, teniendo en cuenta que la exclusión política de amplios sectores sociales y territorios han sido causas estructurales del conflicto armado en Colombia, incidir sobre este tema y profundizar la democracia a nivel nacional y local es fundamental para la construcción de la paz.

\section{Conclusión}

Con el presente trabajo se busca subrayar que un potencial escenario de post conflicto en Colombia encierra profundos desafíos políticos, sociales y económicos. A la firma de un acuerdo de paz seguirán largos años de un complicado y exigente proceso de construcción de paz, que se pretende sea duradera. La sostenibilidad de la paz implica la participación de amplios sectores de la sociedad colombiana y requiere que los acuerdos toquen a las regiones y a las comunidades que vivenciaron décadas de violencia. Es fundamental que la construcción de la paz en el post conflicto encierre un matiz y un enfoque regional, pues Colombia es regionalmente diferenciada, tal como sus instituciones y su conflicto armado. De hecho, el macro conflicto nacional se compone de distintos conflictos a nivel micro. El conflicto armado es desigual y territorializado en sus características y manifestaciones. Cada región desarrolla sus especificidades en términos del conflicto y hay expresiones regionalmente diferenciadas del conflicto. Una política integral y sostenible de paz en el post conflicto tendrá necesariamente que ser consciente de esta realidad.

En este contexto, el potencial del modelo y enfoque de construcción de paz del PDPMM en un escenario de post conflicto es inmenso. Representa un modelo integral y multidimensional de construcción de paz positiva y sostenible desde la base. Abarca elementos que, si se desarrollan en una escala más amplia, pueden construir los cimentos para una paz duradera y positiva en el país. De sus procesos sociales y su enfoque para la paz pueden rescatarse buenas prácticas en términos de construcción de una paz sostenible, fomento de 
desarrollo rural y generación de una presencia integral del Estado en el territorio (Barreto, 2013). Muestra líneas de acción concretas para la construcción de la paz duradera a partir de la especificidad de las regiones y territorios de Colombia, estableciendo una metodología válida de aproximación a las causas del conflicto y de inclusión de grupos sociales tradicionalmente excluidos, como los campesinos y los jóvenes (Barreto, 2012).

Por todos estos elementos, el PDPMM pone de manifiesto que una estrategia sostenible e integral de construcción de la paz se vincula necesariamente con una apuesta de construcción de sociedad, Estado y democracia y con la generación de una cultura de paz, civilidad y diálogo. Estos serán los mayores retos de Colombia en un potencial y deseable escenario de post conflicto.

\section{Bibliografía}

Barreto, M. (2009). El Laboratorio de Paz del Cauca/Nariño: una salida indígena para la paz en Colombia? En Restrepo, J., \& Aponte, D. (Eds.) Guerra y violencia en Colombia: herramientas e interpretaciones (pp. 545-585). Bogotá: Editorial Javeriana.

Barreto, M. (2012). "Laboratorios de Paz" en territorios de violencia(s): ¿abriendo caminos para la paz positiva en Colombia? Tesis de doctorado no publicada. Universidad de Coimbra, Portugal.

Barreto, M. (s.f.). PDPMM. En Experiencias de Paz: lecciones aprendidas para Colombia - Presencia Integral del Estado en el Territorio (PIET) (pp. 36-110). En proceso de publicación. Bogotá: Universidad Jorge Tadeo Lozano/Oficina del Alto Comisionado Para La Paz.

Briceño, L. H. (2007). Finca campesina de ladera en el Magdalena Medio: ocupación productiva del territorio con cacao y frutales. En PNUD. Sistematización de los procesos de desarrollo económico y social adelantados por el PDPMM entre 1996 y 2006 (pp. 139-164). Barrancabermeja: PNUD.

Burton, J. (1990). Conflict Resolution and Provention. New York: St. Martin's Press.

Castilla, P. (2007). Minería, petróleo, energía y multinacionales. Magdalena Medio: generador de energía riqueza nacional. Colombia: Observatorio de Paz Integral del Magdalena Medio, OPI. Recuperado de http://www.opi.org.co/pdfs/ANALISIS\%2OMEGAPROYECTOS.pdf

Corporación Desarrollo y Paz del Magdalena Medio, CDPMM (2005). Programa Laboratorio de Paz en el Magdalena Medio: Plan Operativo Global, II Fase 2005 - 2009. Barrancabermeja: PDPMM. 
Escola de Cultura de Pau, ECP (2006). Construyendo paz en medio de la guerra. Barcelona: ECP. Recuperado de http://escolapau.uab.cat/img/programas/colombia/ colombiao20e.pdf

Galtung, J. (1996). Peace by peaceful means: Peace and Conflict, Development and Civilization, London: Sage Publications.

García, A., \& Sarmiento, A. (2002). Programas Regionales de Desarrollo y Paz: casos de capital social y desarrollo institucional. Bogotá: Fundación Ideas Para la Paz, PNUD.

Granada, S., Restrepo, J., \& Vargas, A. (2009). El agotamiento de la política de seguridad: evolución y transformaciones recientes en el conflicto armado colombiano. En Restrepo, J., \& Aponte, D. (Eds.). Guerra y violencia en Colombia: herramientas e interpretaciones (pp. 27-124). Bogotá: Editorial Javeriana.

Guarín, S. (2008). Potencialidades y límites de la incidencia de las organizaciones de la sociedad civil en la formulación, implementación y seguimiento de las políticas públicas: el caso de los Programas Regionales de Desarrollo y Paz. I Congreso de Ciencia Política, septiembre 30 - octubre 3. Bogotá.

Katz, M. (2004, febrero). Experiencia regional de paz: El Programa de Desarrollo y Paz del Magdalena Medio. Controversia, (no. extrordianario), 30-34. Recuperado de http://www.c-r.org/downloads/Accord14_SPA.pdf

Lederach, J. P. (1997). Building Peace. Sustainable Reconciliation in Divided Societies. Washington: United States Institute of Peace Press.

McDonald, G. (1997). Peacebuilding from below. Alternative perspectives on Colombia's peace process. London: Catholic Institute for International Relations.

Molano, A. (2009). 30 años de construcción de paz en el Magdalena Medio. Bogotá: CINEP, Corporación Colombiana de Proyectos Sociales.

Observatorio de Paz Integral, OPI (2006). Informe Semestral de la situación de violación a los $\mathrm{DDHH}$, violencia política, infracciones al DIH, acciones bélicas y acciones colectivas por la paz en el Magdalena Medio. Barrancabermeja: OPI.

Pécaut, D. (1992). Guerrillas and Violence. En Bergquist, C., Sánchez, G., \& Peñaranda, R. Violence in Colombia: The Contemporary Crisis in Historical Perspective (pp. 214-240). Wilmington: SR Books.

PNUD (2008). Aprendizajes de la estrategia de economía popular urbana. En Rutas, Caminos y Experiencias de nuestra gente del Magdalena Medio (pp. 321-327). Bogotá: PNUD. 
Pureza, J. (2001). Introdução: Estudos sobre a Paz e Cultura da Paz. En Pureza, J. (Ed.). Para uma cultura da paz (pp. 7-28). Coimbra: Quarteto.

Richmond, O. (2008). Peace in Internacional Relations. Londres: Routledge.

Romero, Y., \& Navarro, J. (2010). Análisis de los procesos de presupuesto participativo para los municipios de Bolívar y el Peñón, en el Departamento de Santander y su aporte al ejercicio de la democracia participativa. Bucaramanga: Universidad Industrial de Santander.

Saavedra, M. R., \& Ojeda, L. (2006). Trabajo en Red: Imaginarios conceptuales de paz, desarrollo y región en los programas de la Red Prodepaz. Documentos Ocasionales, (74). Bogotá: CINEP.

Sousa, B. (2003). Democratizar a Democracia: Os Caminhos da Democracia Participativa. Porto: Edições Afrontamento.

Villamarín, R. (2005). Construyendo nación desde la región: La experiencia del Sistema Regional de Planeación Participativa del Magdalena Medio. En Suarez, Ó., Bayona, M., \& Gutiérrez, J. (Eds.). Relatos desde la Memoria: Sistematización de Experiencias del PDPMM. Barrancabermeja: PDPMM. 